\title{
Correlação entre ressonância magnética, expressão do Ki-67 e p53, e prognóstico em astrocitomas
}

\section{Correlation between Magnetic Ressonance Image, Expression of Ki-67 and p53, and Prognosis in Astrocytomas}

Patrícia Maluf Cury ${ }^{1}$ José Roberto Lopes Ferraz Filho ${ }^{2}$ Darlene Arruda ${ }^{3}$ Gibran Franzoni Rufca ${ }^{4}$

Rachel Zerbini ${ }^{5}$
Address for correspondence Gibran Frazoni Rufca, MD, Rua José Justino de Carvalho, 2162, Jardim Matilde, Ourinhos, SP, Brasil, CEP 19901-560 (e-mail: gibran.neuro@terra.com.br).

Trabalho realizado nos Departamentos de Patologia, Radiologia e Neurocirugia do Hospital de Base de São José do Rio Preto (HB) e da Faculdade de Medicina de São José do Rio Preto (Famerp),

\section{Resumo \\ Palavras-Chave \\ - astrocitoma \\ - ressonância magnética \\ - antígeno Ki-67 \\ - proteína supressora tumoral p53 \\ - prognóstico}

Objetivos Avaliar a correlação entre a expressão do Ki-67 e do p53 em astrocitomas graus II a IV, as características da RM e seu prognóstico.

Métodos e Resultados Este é um estudo longitudinal retrospectivo do tipo coorte histórico que avaliou 45 pacientes. O prognóstico foi avaliado mediante revisão dos prontuários. A avaliação imuno-histoquímica foi realizada no Serviço de Patologia do HB. A avaliação das imagens de RM foi feita no Serviço de Radiologia. A sobrevida média dos pacientes foi de 581,4 dias no grau IV; 1.704,8 dias no grau III; e 1.044,8 dias no grau II. Para cada grau, os seguintes percentuais de células positivas de Ki-67 e p53 tiveram respectivamente: 97,8 e $80 \%$ de positividade no grau IV; 72,7 e $73,4 \%$ de positividade no grau III; e 70 e $80 \%$ de positividade no grau II. Observou-se diferença estatística na expressão de Ki-67 entre os graus III e IV $(P=0,014)$. A RM mostrou especificidade de $64 \%$ e sensibilidade de $100 \%$ para a presença de necrose tumoral. Conclusão Concluímos que a análise da positividade do Ki-67 e do p53 não permite sua correlação com a sobrevida dos pacientes, apesar de ambos terem sua frequência distribuída de maneira semelhante. A RM tem especificidade moderada em relação ao exame patológico.
Recebido

August 25, 2014

Aprovado

March 31, 2015
DOI http://dx.doi.org/

10.1055/s-0035-1554045. ISSN 0103-5355.
Copyright $@ 2015$ by Thieme Publicações License terms

Ltda, Rio de Janeiro, Brazil 


Abstract
Keywords
- astrocytoma
- magnetic resonance
imaging
- Ki-67 antigen
- tumor suppressor
protein p53
- prognosis

Objectives To evaluate the correlation between the expression of Ki -67 and p53 in astrocytomas grades II to IV, the characteristics of MRI and its prognosis.

Methods This was a retrospective longitudinal cohort study of its kind that evaluated 45 patients. Prognosis was assessed by review of medical records. The immunohistochemical evaluation was performed at the Pathology Service of HB. The evaluation of MRI images was taken in the Radiology Department. The median survival of patients was 581.4 days in grade IV; 1704.8 days in grade III; 1044.8 days in grade II. For each grade, the following percentages of cells positive for $\mathrm{Ki}-67$ and $\mathrm{p} 53$, respectively : $97.8 \%$ and $80 \%$ had a positive grade IV $; 72.7 \%$ and $73.4 \%$ positivity in grade $I I I ; 70 \%$ and $80 \%$ in stage II positivity. There was statistical difference in Ki -67 between grades III and IV ( $P=0.014)$. MRI showed $64 \%$ specificity and $100 \%$ sensitivity for the presence of tumor necrosis.

Conclusion We conclude that the analysis of the positivity of Ki -67 and p53 expression does not allow correlation with patient survival, although both their frequency distributed similarly. MRI has moderate specificity in relation to pathological examination.

\section{Introdução}

Os gliomas são as neoplasias primárias mais frequentes do sistema nervoso central (SNC), correspondendo a $90 \%$ dos tumores, que compreendem ependimomas, oligodentrogliomas e astrocitomas. $O$ astrocitoma, representando $51,2 \%$ dos gliomas, é o tipo histológico mais frequente, com incidência de 3,81 casos por cem mil habitantes nos Estados Unidos. ${ }^{1}$ Não existem dados oficiais sobre a incidência no Brasil. Estes tumores estão associados a uma sobrevida de $10 \%$ em 6 meses. ${ }^{2}$ A Organização Mundial da Saúde (OMS) classifica atualmente os astrocitomas em quatro categorias: grau I, o astrocitoma pilocítico; grau II, o fibrilar; grau III o anaplásico e grau IV, o glioblastoma multiforme. Os astrocitomas grau I são tumores focais de baixo grau, mais frequentes em crianças. Os graus II a IV são tumores que tendem a se infiltrar difusamente no cérebro. São também divididos pela OMS em astrocitomas primários, aqueles que possuem alto grau no momento do diagnóstico, e secundários, os que progridem de um grau menor para um grau maior de indiferenciação. ${ }^{1,2}$

Para avaliação prognóstica dos pacientes portadores de astrocitomas são aceitos os seguintes fatores: idade no diagnóstico, escala de desempenho de Karnofsky, classificação histológica, presença de sintomas epiléticos, localização do tumor, sucesso da radioterapia e da quimioterapia, tamanho da cirurgia de ressecção do tumor e recorrência após a cirurgia. $^{3-5}$

Entretanto, diversos estudos vêm propondo que técnicas laboratoriais e radiológicas também podem ser utilizadas na avaliação prognóstica. Dentre elas, destacam-se a avaliação de marcadores de proliferação celular e de apoptose, e os exames de ressonância magnética (RM). Quanto maior a proliferação celular e mais inibida a apoptose mais agressivo é o tumor. Os marcadores imuno-histoquímicos mais citados são o Ki-67 para proliferação celular e o p53 para apoptose. ${ }^{5-14}$ Da mesma maneira, as características do tumor no exame de RM mostram que quanto maior a vascularização e a capilaridade mais avançado é o tumor. ${ }^{15-18}$

O antígeno Ki-67 é uma proteína, cujo gene é localizado no cromossomo 10, expressada em todas as fases do ciclo celular (G1-S-G2-M), exceto em G0. Sua expressão é regulada por cascatas proteolíticas. Portanto, é estritamente associada à proliferação celular e muito usada na patologia como marcador de proliferação celular. Entretanto, sua função ainda permanece desconhecida. ${ }^{19}$

O p53 é uma proteína codificada por um gene situado no cromossomo 17. Sua principal função está relacionada à preservação da integridade do código genético em cada célula ao longo de toda a molécula de DNA. Durante o ciclo de divisão celular, a proteína p53 faz uma verificação quanto à presença de um erro de replicação. Caso seja verificada a existência deste o p53 impede que esta célula entre em processo de mitose e complete a divisão celular. Para isto, dois caminhos poderão ser seguidos: a correção da mutação através da ativação de proteínas de reparo ou a indução da morte celular por apoptose. A relação entre a proteína p53 e a carcinogenese tem sido amplamente comprovada através do elevado índice de mutações de seu gene em tumores malignos de diferentes tecidos do organismo. ${ }^{20}$

Shiraishi et al., Aldape et al. e Tortosa et al. encontraram uma maior incidência de mutação do p53 em astrocitomas do que em glioblastomas sem diferença estatística significante de prognóstico entre os pacientes com esta mutação. 13,21,22 Já Birner et al., Ribeiro et al. e Korschunov et al. encontraram que a expressão da proteína p53 relaciona-se com uma maior sobrevida dos pacientes. ${ }^{12,23}$

Faria et al. observaram um aumento da expressão do Ki-67 de acordo com o grau do astrocitoma. ${ }^{9}$ Moskowitz et al., Tortosa et al. e Kato et al. mostraram que a expressão do Ki-67 não foi preditora de maior sobrevivência. ${ }^{6,8,21}$ Já Johannessen et al. e Uematsu et al. demonstraram que este marcador pode ser um importante fator prognóstico clínico de astrocitomas. ${ }^{5,7}$ 
A RM com imagens nas ponderações T1 e T2 são frequentemente utilizadas no diagnóstico de lesões encefálicas de diversas etiologias. ${ }^{24} \mathrm{O}$ uso do contraste paramagnético no exame de RM encefálica pode auxiliar na caracterização do grau de agressividade das lesões neoplásicas, fornecendo informações do padrão de captação do contraste, mostrando integridade ou quebra da barreira hematoencefálica, edema perilesional, lesão tumoral à distância, hemorragia, necrose $\mathrm{e}$ efeito de massa, apesar de existirem controvérsias entre informações obtidas por este método de imagem e os resultados anatomopatológicos. ${ }^{17,23}$

Segundo Meng et al., a presença de quebra de barreira hematoencefálica pode estar associada a neoplasias de alto grau. ${ }^{17} \mathrm{~A}$ hiperintensidade perilesional nas sequências ponderadas em T2 em imagens de RM convencional na ponderação T2 também não é específica, podendo representar infiltração tumoral, edema vasogênico ou ambos. Com relação ao efeito de massa e à presença de necrose, Meng et al. ressaltaram que esses parâmetros são os fatores preditivos mais importantes para o grau tumoral, porém não são específicos. $^{17}$

Piarillini et al. mostraram que a extensão da necrose foi a única característica radiológica que se correlacionou diretamente com a sobrevivência dos pacientes com glioblastoma multiforme. ${ }^{16}$ Plathow et al. mostraram que o edema perilesional na ponderação T2 e multifocalidade do realce pelo contraste não foram indicadores prognósticos. ${ }^{18}$ Demonstraram, porém, que a ausência de realce pelo contraste na RM correlaciona-se com pior prognóstico dos pacientes. Pope et al. evidenciaram que tumores sem realce ao contraste, edema e multifocalidade das lesões foram indicadores de mau prognóstico dos pacientes. ${ }^{15}$

Devido ao fato de existirem dados conflitantes na literatura atual quanto à relação entre estes dois marcadores, as características da RM e o comportamento tumoral, este estudo tem como objetivo avaliar a correlação entre a expressão do Ki-67 e do p53 em amostras de astrocitomas humanos graus II a IV, as características da RM e o prognóstico dos pacientes.

\section{Metodologia}

Este estudo avaliou 40 pacientes com diagnóstico de astrocitoma grau II, III e IV, divididos em três grupos de 15, 15 e 10 pacientes, respectivamente, em acompanhamento no serviço de neurocirurgia do Hospital de Base de São José do Rio Preto, no período de dezembro de 2006 a janeiro de 2002, quando se iniciaram os estudos de ressonância magnética neste hospital. Os pacientes selecionados realizaram exame de ressonância magnética encefálica e biópsia da neoplasia com estudo anatomopatológico. Foram incluídos pacientes independentemente de idade, sexo, comorbidades e tratamento.

O prognóstico foi avaliado mediante revisão dos prontuários dos pacientes. A avaliação imuno-histoquímica foi realizada no Serviço de Anatomia Patológica do Hospital de Base de São José do Rio Preto, por bióloga experiente (Eliane Keid Martins). A avaliação das imagens de RM foi feita por neuroradiologista experiente no Serviço de Imagem e Radiologia do Hospital de Base de São José do Rio Preto (Prof. Dr. José Roberto Ferraz).

\section{Imuno-histoquímica}

A avaliação das proteínas p53 e Ki-67 foi realizada com o auxílio da técnica imuno-histoquímica de estreptavidinabiotina-peroxidase, acompanhada da análise quantitativa dos elementos positivos através de contagem manual.

Todas as amostras utilizadas nas reações imuno-histoquímicas foram fixadas em formol a $10 \%$ e emblocadas em parafina. A reação imuno-histoquímica foi desenvolvida sobre secções teciduais de $4 \mu \mathrm{m}$ de espessuras estendidas em lâminas de vidro, previamente limpas e desengorduradas, e preparadas com adesivo à base de (3-Aminopropyl) triethoxysilane (Sigma-Aldrich Co., St. Louis, MO, EUA).

As lâminas foram colocadas na estufa a $60^{\circ} \mathrm{C}$, durante 30 minutos, e para desparafinar, as amostras foram imersas no xilol a frio em temperatura ambiente, por 30 minutos (dois banhos de 15 minutos, em dois recipientes com xilol).

As secções teciduais foram submetidas, em seguida, ao tratamento em panela a vapor para recuperação antigênica. Este passo foi realizado através da colocação das amostras histológicas dentro da cuba, aquecendo a solução recuperadora composta de ácido cítrico (pH 6) 2,1 g e $1.000 \mathrm{~mL}$ de água destilada até atingir a temperatura de $96^{\circ} \mathrm{C}$ na panela a vapor; ao atingir esta temperatura, as lâminas foram colocadas dentro da cuba com a solução, deixando aquecer por 28 minutos. Depois do tratamento e da cuba com a solução recuperadora, foram colocadas dentro de outra vasilha, onde ficarão resfriando por 30 minutos fora da panela.

Seguiu-se, então, o bloqueio de peroxidase endógena tecidual, através da imersão das lâminas durante 30 minutos (temperatura ambiente) em uma cuba com água oxigenada a 3\% (30 volumes) diluída em metanol. Após este passo, em todos os procedimentos de lavagem a seguir, as lâminas foram lavadas com a solução tampão PBS (Phosphate Buffered Saline).

Em seguida, as secções teciduais foram submetidas à incubação dos anticorpos primários: antígeno Ki-67, mouse monoclonal antibody, clone MM1 (Novocastra, Leica Biosystems Newcastle, Reino Unido) diluído 1:400 e proteína p53, mouse monoclonal antibody, clone DO-7 (Novocastra, Leica Biosystems Newcastle, Reino Unido), diluído 1:200. Ambos foram aplicados com pipeta, uma quantidade de $100 \mu \mathrm{L}$ sobre a amostra na lâmina. $\mathrm{O}$ anticorpo primário será diluído em solução tampão contendo albumina sérica bovina a $1 \%$, diluída em PBS. As lâminas com todos os anticorpos serão colocadas durante 18 horas (overnight) a $4^{\circ} \mathrm{C}$.

Após três lavagens em PBS, cada lavagem durando 5 minutos, os cortes receberão o anticorpo secundário biotinilado. Foi utilizado o anticorpo secundário polivante (anticabra, anticoelho, anticamundongo). Foi utilizado o Universal Large Volume DAKO LSAB ${ }^{\circledR}$ (labelled streptavidin biotin) + Kit, Peroxidase (DakoCytomation, Carpinteria, CA, EUA). Foram aplicados $100 \mu \mathrm{L}$ de solução A nas amostras por 30 minutos, lavando três vezes em PBS por 5 minutos cada. Posteriormente, foi aplicada a solução B por mais 30 minutos, 
repetindo o mesmo processo de lavagem da solução A. O redor da amostra foi enxugado com papel absorvente.

Para a revelação da reação foi utilizada solução cromógena constituída de $60 \mathrm{mg}$ de diaminobenzidina (3,3'-Diaminobenzidine tetrahydrochloride, Sigma-Aldrich Co., St. Louis, MO, EUA) diluída em $100 \mathrm{~mL}$ de PBS, e esta solução foi dissolvida em $300 \mathrm{~mL}$ de $\mathrm{H}_{2} \mathrm{O}_{2}$ (10 volumes). As lâminas ficaram mergulhadas na solução de 3 a 5 minutos em uma estufa a $37^{\circ} \mathrm{C}$ em câmara escura. Em seguida foram lavadas em água destilada.

Os cortes foram contracorados com hematoxilina de Harris por 15 minutos e lavados novamente, para a remoção do excesso. Após desidratação e diafanização, a montagem das lâminas foi realizada com Sparlack (Verniz Ypiranga, Brasil), verniz cristal brilhante e lamínula. Os controles positivos foram realizados para p53 e para Ki-67. Os controles negativos não foram realizados, visto que não haveria necessidade destes com o controle positivo estando marcado.

\section{Análise das Reações Imuno-histoquímicas}

As lâminas preparadas para imuno-histoquímica tiveram as áreas dos cortes selecionadas para contagem demarcadas previamente, garantindo que a análise da região escolhida foi realizada no mesmo local escolhido em $\mathrm{H} \& \mathrm{E}$.

Os procedimentos de contagem foram feitos por um observador utilizando um microscópio de luz Olympus modelo CX40RF100 (Japão) no aumento final de 400x.

Foram contadas as células positivas para cada um dos antígenos pesquisados, sempre que possível, em um total de quinhentas células da lesão. 0 processo de contagem foi realizado através do software Adobe Photoshop Extended CS3@ para Windows XP e Vista.

\section{Estudo da RM}

Os exames de ressonância magnética (RM) foram realizados em um aparelho Phillips Gyroscan Intera de 1,5 tesla, utilizando bobina de cabeça.

As imagens foram obtidas na ponderação FAIR utilizando TE 120 , TR 6000 , FOV $250 \times 80$, matriz $256 \times 512$, NSA 3, espessura de corte de $5 \mathrm{~mm}$ com gap de $1 \mathrm{~mm}$; sequência turbo spin-eco ponderação T2 no plano axial com TE 110, TR 4466 , FOV $250 \times 80$, matriz $400 \times 512$, NSA 2, espessura de corte de $5 \mathrm{~mm}$ com gap de $1 \mathrm{~mm}$; sequência difusão no plano axial com TE 86, TR 1000 , FOV $230 \times 100$, matriz $128 \times 512$, NSA 1, espessura de corte de $5 \mathrm{~mm}$ e gap de $1 \mathrm{~mm}$; sequência eco-planar ponderação T2 no plano axial com TR 994, TE 40, FOV 270, matriz $256 \times 512$, NSA 4, espessura de corte de $5 \mathrm{~mm}$ com gap de $1 \mathrm{~mm}$; sequência spin-eco ponderação T1 nos planos axial, coronal e sagital pré e pós infusão do meio de contraste paramagnético com TE 15, TR 550, FOV $230 \times 85$, matriz $256 \times 512$, NSA 2 , espessura de corte de $5 \mathrm{~mm}$ com gap de $1 \mathrm{~mm}$.

Foram estudadas as seguintes características tumorais à RM:

- realce ao contraste;

- necrose;

- edema;
- hemorragia;

- tipo de lesão.

\section{Análise estatística}

A análise estatística foi realizada utilizando-se testes não paramétricos: medianas, médias, desvio padrão, ANOVA, regressão logística, estatística tabulada, Kaplan-Meier e Cox, análise de curva ROC (característica de operação do receptor) com nível de significância menor ou igual a $5 \%$. Foram utilizados os softwares GraphPad Prism@ versão 4.00 para Windows (GraphPad Software, San Diego, Califórnia, EUA) e MedCalc@ versão 9.5.1.0 para Windows.

\section{Resultados}

Do total $(\mathrm{n}=40), 27,5 \%$ eram do sexo feminino e $72,5 \%$ eram do sexo masculino; quanto à distribuição etária, a média de idade para cada grupo foi: $56,60( \pm 16,15)$ anos para o grau IV, 46,47 ( $\pm 17,96)$ anos para o grau III; 45,92 ( $\pm 12,75)$ anos para o grau II.

Quanto à sobrevida média por grupo, obtivemos 0,92 ano para o grau IV; 2,60 anos para o grau III; 2,16 anos para o grau II. A sobrevida em 2 anos de cada faixa etária foi de $50 \%$ para os pacientes de zero a 20 anos; $87,5 \%$ para 21 a 40 anos; $43 \%$ para 41 a 60 anos; $55 \%$ para 61 a $80 \%$; com p $=0,6589$.

De acordo com a porcentagem de positividade Ki-67, quando esta é maior que 5\%, a sobrevida em 2 anos foi de $47 \%$, e quando é menor que $5 \%$, foi de $48 \%$, com $\mathrm{p}=0,6631$ (-Fig. 1). Com relação ao p53, quando é maior que $5 \%$, a sobrevida em 2 anos foi de $35 \%$, e quando é menor que $5 \%$, foi de $60 \%$, com $\mathrm{p}=0,5164$.

Encontramos que 75,3\% dos pacientes grau IV apresentam positividade maior que $5 \%$ de $\mathrm{Ki}-67$, e $66,6 \%$ positividade maior que $5 \%$ de $\mathrm{p} 53 ; 26,6 \%$ dos pacientes grau III apresentam positividade maior que $5 \%$ de Ki-67, e $40 \%$ positividade maior que $5 \%$ de $\mathrm{p} 53 ; 60 \%$ dos pacientes grau II apresentam positividade maior que $5 \%$ de $\mathrm{Ki}-67$, e $90 \%$ positividade maior que $5 \%$ de p53.

Quando a porcentagem de positividade Ki-67 é maior que 5\%, a sobrevida em 2 anos, por grupo, é de: $50 \%$ para o grau IV;

\section{Sobrevida de acordo com Ki67}

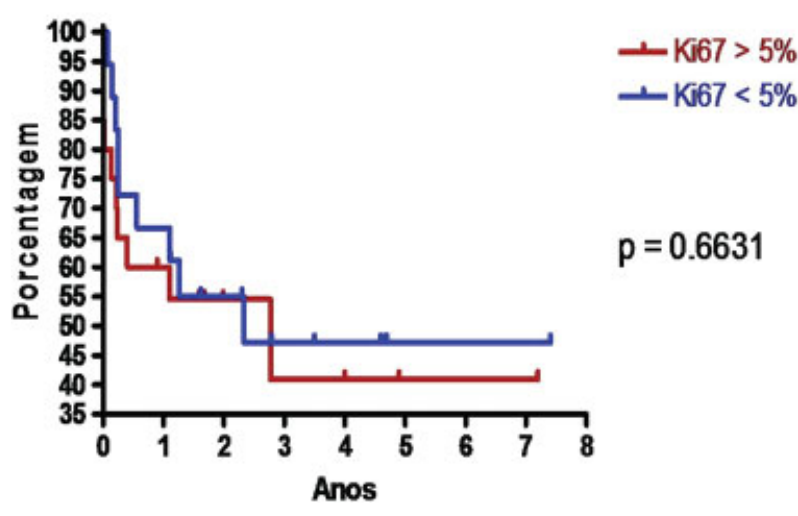

Fig. 1 Sobrevida de acordo com Ki-67. 
$25 \%$ para o grau III; $80 \%$ para o grau II; com $\mathrm{p}=0,3435$. Quando é menor que 5\%, temos: zero para o grau IV; 60\% para o grau III; $25 \%$ para o grau II; com p = 0,0879; entretanto, no teste log-rank para tendência, obtivemos um $\mathrm{p}=0,0449$, que mostra ser possível pressupor a associação entre Ki-67 menor que $5 \%$ e menor sobrevida.

Já em relação ao p53, quando é maior que 5\%, temos a sobrevida em 2 anos, por grupo, é de: $37 \%$ para o grau IV; $50 \%$ para os graus III e II; com $\mathrm{p}=0,1959$. Quando é menor que $5 \%$, temos: zero para o grau IV; $50 \%$ para o grau III; zero para o grau II; com $\mathrm{p}=0,552$.

Sobre os parâmetros avaliados na RM, obtivemos que a sobrevida em 2 anos foi de: zero na presença de realce ao contraste e de $45 \%$ na sua ausência, com $p=0,3994$; de $40 \%$ na presença de necrose e de $80 \%$ na sua ausência, com $\mathrm{p}=0,0494 ; 25 \%$ com edema e de $50 \%$ sem edema, com $\mathrm{p}=0,0064 ; 28 \%$ na presença de hemorragia e de $25 \%$ na sua ausência, com $\mathrm{p}=0,5110 ; 29 \%$ nas lesões infiltrativas e 39\% nas nodulares, com $\mathrm{p}=0,424$. No caso de análise da relação entre edema e sobrevida, obtivemos um risco relativo (RR) de 2,48 com intervalo de confiança de $95 \%$ [2,350 - 2,614].

Quanto à presença de recidiva tumoral e sua relação com os marcadores imuno-histoquímicos, temos:

- no grau IV, recidiva em $80 \%$ dos casos de Ki-67 maior que 5\%; 53,3\% dos casos de Ki-67 menor que 5\%; 53,3\% dos casos de p53 maior que 5\%; 20\% dos casos de p53 menor que $5 \%$;

- no grau III, recidiva em $6 \%$ dos casos de Ki-67 maior que 5\%; 20\% dos casos de Ki-67 menor que 5\%; 6\% dos casos de p53 maior que 5\%; 20\% dos casos de p53 menor que 5\%;

- no grau II, recidiva de $20 \%$ dos casos de Ki-67 maior que 5\%; 30\% dos casos de Ki-67 menor que 5\%; 40\% dos casos de p53 maior que 5\%; 10\% dos casos de p53 menor que $5 \%$.

Em todos os casos o valor de p obtido através do teste quiquadrado foi igual a 1,000 .

Analisando os testes de Ki-67 e p53 através de curvas ROC para predileção de mau prognóstico, obtivemos para o ponto de corte de $5 \%$ uma sensibilidade e uma especificidade, respectivamente, de 52,63 e $47,37 \%$ para o $\mathrm{Ki}-67$, e de $68 \%$ e $47,37 \%$ para o $\mathrm{p} 53$. Entretanto, obtivemos para um ponto de corte de 5,95\% do Ki-67 uma sensibilidade de 52,63\% e especificidade de $63,16 \%$, e para um ponto de corte de $8,6 \%$ do p53 uma sensibilidade de 57,9\% e especificidade de $63,4 \%$ (-Figs. 2 e 3 ).

\section{Discussão}

Nosso estudo demonstrou que as presenças de necrose e de edema, sinalizadas no exame de RM, foram fatores de menor sobrevida dos gliomas, enquanto a expressão imuno-histoquímica de p53 e Ki-67 não demonstrou relação com o prognóstico. Os outros fatores estudados, como realce ao contraste, hemorragia, tipo de lesão e grau histológico também não apresentaram significado em relação à sobrevida dos doentes.

A expressão de Ki-67 nos tumores não apresentou relação prognóstica quanto à sobrevida quando analisada na amostra total e por grupos. Entretanto, é possível supor associação

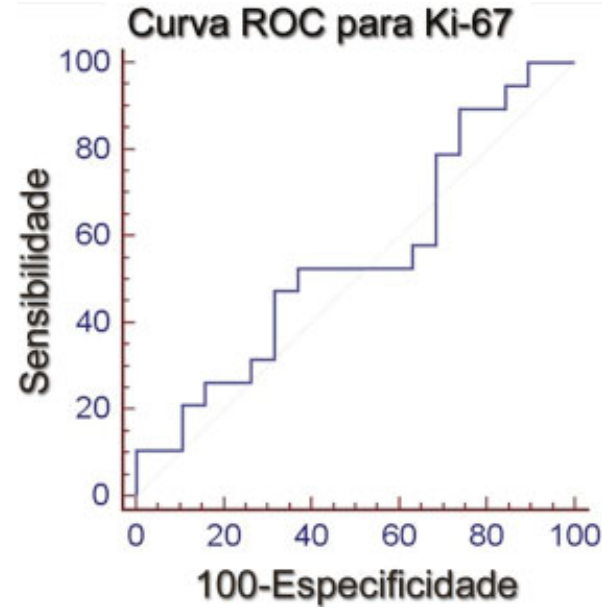

Fig. 2 Curva ROC (característica de operação do receptor) para Ki-67.

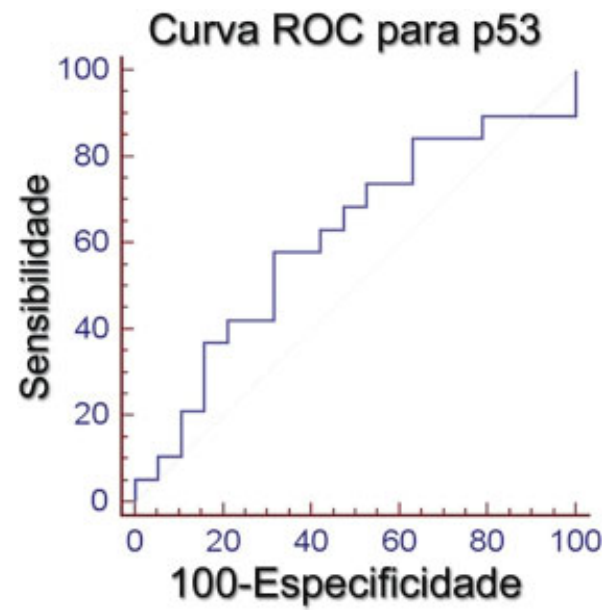

Fig. 3 Curva ROC (característica de operação do receptor) para p53.

entre Ki-67 menor que 5\% e menor sobrevida, estando de acordo com Moskowitz et al., Tortosa et al. e Kato et al., os quais mostraram que a expressão do Ki-67 não foi preditora de maior sobrevivência, pois encontramos no teste log-rank para tendência um $\mathrm{p}=0,0449$, mostrando a associação entre Ki-67 menor que 5\% e menor sobrevida. ${ }^{6,8,21}$ Já Johannessen et al. e Uematsu et al. demonstraram que este marcador pode ser um importante fator prognóstico clínico de astrocitomas. ${ }^{5,7}$ Faria et al. observaram um aumento da expressão do Ki-67 de acordo com o grau do astrocitoma. ${ }^{9}$

Assim como o Ki-67, o marcador p53 não teve relação prognóstica nas amostras total e de cada grupo, concordando com Shiraishi et al., Aldape et al. e Tortosa et al. que encontraram maior incidência de mutação do p53 em astrocitomas do que em glioblastomas, não havendo diferença estatística significante de prognóstico entre os pacientes com esta mutação. ${ }^{13,21,22}$ Já Birner et al., Ribeiro et al. e Korschunov et al. encontraram que a expressão da proteína p53 relaciona-se com maior sobrevida dos pacientes. ${ }^{12,23,25}$ 
Quanto ao estudo de RM, encontramos que apenas a necrose foi fator de menor sobrevida e que o edema foi fator de risco para pior prognóstico com RR de 2,4, assim como Piarillini et al. e Pope et al. mostraram que a necrose, edema e multifocalidade das lesões foram indicadores de mau prognóstico dos pacientes. ${ }^{15,16}$ Entretanto para os outros parâmetros radiológicos avaliados não foi possível correlacionálos com a sobrevida dos casos, estando parcialmente de acordo com Meng et al., cujos resultados mostraram que apenas o efeito de massa e a presença de necrose foram fatores preditivos mais importantes para o grau tumoral, porém, não específicos, e com Plathow et al., que encontraram o edema perilesional na ponderação T2 e multifocalidade do realce pelo contraste como não indicadores prognósticos, porém, demonstraram que a ausência de realce pelo contraste na RM correlaciona-se com pior prognóstico dos pacientes. ${ }^{17,18}$

Interessantemente, embora em nosso estudo a presença de necrose na RM tenha-se correlacionado com a sobrevida, não observamos diferença de prognóstico quando comparamos os gliomas grau III e IV. Isto pode ser devido ao pequeno número de casos por grupo, ou ainda por questão de amostragem do material, já que a presença de necrose no exame histológico classifica a neoplasia em grau IV.

O tamanho da amostra também pode ter prejudicado as outras correlações, pois ao observarmos os gráficos da curva ROC notamos uma aparente diferença entre os grupos, tanto em relação ao prognóstico dos tipos histológicos, como quanto à expressão imuno-histoquímica. Um maior número de casos poderia talvez evidenciar uma correlação estatisticamente significativa.

O número pequeno de casos foi devido ao interesse de avaliar a correlação entre os achados histológicos e imunohistoquímicos com os achados imaginológicos da RM. Com isso, pudemos observar a necessidade de uma amostra adequada, retirada do local apropriado, para poder realizar o diagnóstico correto do grau da lesão.

\section{Conclusão}

Diante do exposto, não foi possível estabelecer relação entre o prognóstico e a análise imuno-histoquímica do Ki-67 e p53. Acreditamos que é necessário um estudo com uma maior amostra para melhor avaliação.

No estudo da RM foi possível estabelecer relação entre a presença de necrose e menor sobrevida. Quanto à presença de edema, apesar de um valor de p no estudo de Kaplan Meier não significativo, obtivemos um risco relativo de $2,4 \mathrm{com}$ intervalo de confiança significativo, mostrando que o edema pode ser fator de risco para maior mortalidade. Em relação aos outros critérios de imagem, não foi possível estabelecer relação significativa. A relação de recidiva com Ki-67 e p53 também não foi significativa.

Através das curvas ROC obtivemos para o ponto de corte de $5 \%$ uma sensibilidade e uma especificidade, respectivamente, de 52,63 e $47,37 \%$ para o Ki-67, e de 68 e $47,37 \%$ para o p53. Entretanto, sugerimos um ponto de corte de 5,95\% para Ki-67 com sensibilidade de 52,63\% e especificidade de
$63,16 \%$, e um ponto de corte de $8,6 \%$ para p53 com sensibilidade de $57,9 \%$ e especificidade de $63,4 \%$.

\section{Referências}

1 Kleihues P, Louis DN, Scheithauer BW, et al. The WHO classification of tumors of the nervous system. J Neuropathol Exp Neurol 2002;61(3):215-225, discussion 226-229

2 Samuels MA. Update in neurology. Ann Intern Med 2007;146(2): 128-132

3 Mineo JF, Bordron A, Baroncini M, et al. Prognosis factors of survival time in patients with glioblastoma multiforme: a multivariate analysis of 340 patients. Acta Neurochir (Wien) 2007; 149(3):245-252, discussion 252-253

4 Hulshof MC, Koot RW, Schimmel EC, Dekker F, Bosch DA, González González D. Prognostic factors in glioblastoma multiforme. 10 years experience of a single institution. Strahlenther Onkol $2001 ; 177(6): 283-290$

5 Johannessen AL, Torp SH. The clinical value of Ki-67/MIB-1 labeling index in human astrocytomas. Pathol Oncol Res 2006; 12(3):143-147

6 Moskowitz SI, Jin T, Prayson RA. Role of MIB1 in predicting survival in patients with glioblastomas. J Neurooncol 2006; 76(2):193-200

7 Uematsu M, Ohsawa I, Aokage T, et al. Prognostic significance of the immunohistochemical index of survivin in glioma: a comparative study with the MIB-1 index. J Neurooncol 2005;72(3): 231-238

8 Kato H, Fujimura M, Kumabe T, Ishioka C, Kanamaru R, Yoshimoto T. PTEN gene mutation and high MIB-1 labeling index may contribute to dissemination in patients with glioblastoma. J Clin Neurosci 2004;11(1):37-41

9 Faria MHG, Gonçalves BP, do Patrocínio RM, de Moraes-Filho MO, Rabenhorst SHB. Expression of Ki-67, topoisomerase Ilalpha and c-MYC in astrocytic tumors: correlation with the histopathological grade and proliferative status. Neuropathology 2006;26(6): 519-527

10 Lindboe CF, von der Ohe G, Torp SH. Determination of proliferation index in neoplasms using different Ki-67 equivalent antibodies. APMIS 2003;111(5):567-570

11 Simmons ML, Lamborn KR, Takahashi M, et al. Analysis of complex relationships between age, p53, epidermal growth factor receptor, and survival in glioblastoma patients. Cancer Res 2001;61(3): 1122-1128

12 Birner P, Piribauer M, Fischer I, et al. Prognostic relevance of p53 protein expression in glioblastoma. Oncol Rep 2002;9(4): 703-707

13 Shiraishi S, Tada K, Nakamura H, et al. Influence of p53 mutations on prognosis of patients with glioblastoma. Cancer 2002;95(2): 249-257

14 Lutterbach J, Sauerbrei W, Guttenberger R. Multivariate analysis of prognostic factors in patients with glioblastoma. Strahlenther Onkol 2003;179(1):8-15

15 Pope WB, Sayre J, Perlina A, Villablanca JP, Mischel PS, Cloughesy TF. MR imaging correlates of survival in patients with high-grade gliomas. AJNR Am J Neuroradiol 2005;26(10):2466-2474

16 Pierallini A, Bonamini M, Pantano P, et al. Radiological assessment of necrosis in glioblastoma: variability and prognostic value. Neuroradiology 1998;40(3):150-153

17 Law M, Yang S, Wang $\mathrm{H}$, et al. Glioma grading: sensitivity, specificity, and predictive values of perfusion MR imaging and proton MR spectroscopic imaging compared with conventional MR imaging. AJNR Am J Neuroradiol 2003;24(10):1989-1998

18 Plathow C, Lichy MP, Bachert P, Zuna I, Kauczor HU. The role of MRI in patients with astrocytoma WHO II treated with fractionated stereotactic radiotherapy. Eur Radiol 2004;14(4):679-685 
19 Schlüter C, Duchrow M, Wohlenberg C, et al. The cell proliferationassociated antigen of antibody Ki-67: a very large, ubiquitous nuclear protein with numerous repeated elements, representing a new kind of cell cycle-maintaining proteins. J Cell Biol 1993; 123(3):513-522

20 Pinho MSL. Proteína P53: Algum valor clínico ou apenas pesquisa? Uma revisão da literatura. Revista SBCP. Disponível em: http:// www.sbcp.org.br/revista/nbr204/P258_260.htm Acessado em 2014 (Abril 12).

21 Tortosa A, Viñolas N, Villà S, et al. Prognostic implication of clinical, radiologic, and pathologic features in patients with anaplastic gliomas. Cancer 2003;97(4):1063-1071
22 Aldape KD, Okcu MF, Bondy ML, Wrensch M. Molecular epidemiology of glioblastoma. Cancer J 2003;9(2):99-106

23 Ribeiro MdeC, Coutinho LMB, Hilbig A. The role of apoptosis, cell proliferation index, bcl-2, and p53 in glioblastoma prognosis. Arq Neuropsiquiatr 2004;62(2A):262-270

24 Wu WC, Chen CY, Chung HW, Juan CJ, Hsueh CJ, Gao HW; Wen Chu Wu. Discrepant MR spectroscopic and perfusion imaging results in a case of malignant transformation of cerebral glioma. AJNR Am J Neuroradiol 2002;23(10):1775-1778

25 Korshunov A, Golanov A, Sycheva R. Immunohistochemical markers for prognosis of cerebral glioblastomas. J Neurooncol 2002; 58(3):217-236 